\title{
Effect of Body Lotions on Capillary Blood Glucose Measurement - Interference of Hydroquinone-containing Body Lotion with Capillary Glucose Measurement
}

\author{
Andrea MA Omengue, ${ }^{1,2}$ Eugène Sobngwi, ${ }^{1,2,3}$ Mesmin Dehayem, ${ }^{1,2}$ Eric V Balt, ${ }^{4,5}$ Anne MO Boli, ${ }^{1,2}$ Simeon P Choukem, ${ }^{6,7}$ Jean F Gautier ${ }^{8,9}$ \\ and Jean-Claude Mbanya1,2,3 \\ 1. National Obesity Centre, Yaoundé Central Hospital, Yaoundé, Cameroon; 2. Department of Internal Medicine, Faculty of Medicine and Biomedical Sciences, \\ University of Yaoundé 1, Yaoundé, Cameroon; 3. Laboratory of Molecular Medicine and Metabolism, Biotechnology Centre, University of Yaoundé 1, Yaoundé, \\ Cameroon; 4. Diabetes Research Centre, Vrije Universiteit Brussel, Brussels, Belgium; 5. Diabetes Clinic, Department of Internal Medicine, Universitair \\ Ziekenhuis Brussel - UZ Brussel, Vrije Universiteit Brussel, Brussels, Belgium; 6. Faculty of Health Sciences, University of Buea, Cameroon; 7. Department of \\ Internal Medicine, Douala General Hospital, Cameroon; 8. Department of Endocrinology and Diabetes, Lariboisière Hospital, University Paris-Diderot, Paris, \\ France; 9. Inserm UMRS 1138, Cordeliers Research Centre, Paris, France
}

$\mathrm{T}$ he reliability of capillary blood glucose measurements is tremendously important for patients' care and follow-up. Some factors independent of glucose control could however affect readings during ambulatory monitoring of capillary glucose levels in patients with diabetes mellitus. We sought to evaluate the impact of three body lotions commonly used in Cameroon on different strata of glycaemia. We explored their influence over time on measured capillary glucose values. We enrolled 16 participants. Eligible individuals were adult patients with diabetes $(n=12)$ stratified into three levels of capillary glucose values (100 $\pm 40 \mathrm{mg} / \mathrm{dL}[5.55 \pm 2.22 \mathrm{mmol} / \mathrm{L}]$, $200 \pm 40 \mathrm{mg} / \mathrm{dL}[11.1 \pm 2.22 \mathrm{mmol} / \mathrm{L}]$ and $300 \pm 40 \mathrm{mg} / \mathrm{dL}[16.65 \pm 2.22 \mathrm{mmol} / \mathrm{L}])$ and normoglycaemic individuals $(\mathrm{n}=4)$. We measured capillary blood glucose before application, immediately after, then 5, 30 and 60 minutes after application of sweet almond oil, corticosteroid cream and hydroquinone lotion. The measurements made on impregnated body lotion-permeated fingers were compared to that of a clean finger. We observed a significant increase (delta [95\% confidence interval, Cl]: 119.5\% [77.4-222.1]) of capillary glucose level immediately after administration of hydroquinone-containing body lotion ( $p<0.001)$. Capillary glucose values after the use of corticoid cream and sweet almond oil was stable 5, 30 and 60 minutes after application ( $p=0.875$ and $p=0.883$ respectively). In the case of the hydroquinone-containing body lotion, there was a significant difference between capillary glucose level at 5 minutes (delta [95\%Cl]: 81.6\% [55.3-214.2]; $p<0.001$ ), 30 minutes (delta [95\% Cl]: 71.6\% [21.8-134.6]; $\mathrm{p}<0.001$ ) and 60 minutes (delta [95\% Cl]: 58.3\% [2.8-133.3]; $\mathrm{p}=0.013$ ) after application compared to the value obtained from the clean finger. We observed from our study that there were significant variations in capillary blood glucose measurements induced by the use of hydroquinone lotion.

\section{Keywords}

Hydroquinone, body lotions, capillary blood glucose, ambulatory glucose monitoring, self-monitoring of blood glucose

Disclosures: Andrea MA Omengue, Eugène Sobngwi, Mesmin Dehayem, Balti V Eric, Anne MO Boli, Simeon P Choukem, Jean F Gautier and Jean-Claude Mbanya have nothing to declare in relation to this article.

Review Process: Double-blind peer review.

Acknowledgements: The authors acknowledge Dr Noubiap Jean Jacques for editorial assistance. They acknowledge the staff of the National Obesity Centre of the Yaoundé Central Hospital. They are also are grateful to all the patients who participated in the study.

Compliance with Ethics: Procedures were followed in accordance with the Faculty of Medicine and Biomedical Sciences Ethics Committee, University of Yaoundé 1 and with the Helsinki Declaration of 1975 and subsequent revisions, and informed consent was received from all patients involved in the study.

Authorship: All named authors meet the International Committee of Medical Journal Editors (ICMJE) criteria for authorship of this manuscript, take responsibility for the integrity of the work as a whole, and have given final approval to the version to be published.

open Access: This article is published under the Creative commons

Attribution Noncommercial License, which permits any non-commercial

use, distribution, adaptation and reproduction provided the original author(s)

and source are given appropriate credit. (c) The Authors 2018.

Received: 24 January 2018

Accepted: 19 March 2018

Citation: European Endocrinology. 2018;14(1):44-46

Corresponding Author: Eugene Sobngwi, National Obesity Centre, Yaoundé Centra Hospital and Department of Internal Medicine, Faculty of Medicine and

Biomedical Sciences, University of Yaoundé 1, Yaoundé, Cameroon P.O. Box 7535 Yaoundé Cameroon. E: sobngwieugene@yahoo.fr

Support: No funding was received in the publication of this article.
Diabetes mellitus is a major global health problem owing to its increasing prevalence, morbidity and mortality. Blood glucose control is paramount in patients with diabetes to optimise treatment and therefore delay the onset of acute and chronic complications and subsequent death. Capillary blood glucose measurement is the commonly used method to reflect daily glycaemic control in patients with diabetes mellitus. ${ }^{1}$ It is a useful and relatively accessible tool to evaluate and adapt therapy with the aim to achieve optimal glycaemic control. ${ }^{2}$ In patients under continuous subcutaneous insulin infusion (CSII) or multiple daily insulin (MDI) injections, self-monitoring of blood glucose (SMBG) increases patients' awareness of hypoglycaemia and provides strategies to improve prevention of both hypoglycaemic and hyperglycaemic events., ${ }^{3.4}$ However, the accuracy of point-of-care devices in the evaluation of capillary glucose values can be impacted by a number of factors, including the characteristics of the device and the skills of the involved patients with diabetes. ${ }^{5}$ Other factors, such as blood sample, strips, devices, environmental parameters (altitude and/or temperature) and patient behaviours, are linked to SMBG and may impact yield of capillary glucose readings. ${ }^{6.7}$ Self-monitoring of blood glucose often has significant errors that are poorly understood by patients and health care providers, particularly the effect of some substances such as sugary fruits ${ }^{8}$ and body lotions. ${ }^{9}$

We hypothesised that the use of body lotion could significantly influence the results of capillary glycaemia. We therefore sought to evaluate the impact of three body lotions commonly used in Cameroon on different 
strata of glycaemia and we explored their influence over time on measured capillary glucose values.

\section{Methods}

Study design and setting

Study population

We enrolled 16 participants attending the National Obesity Centre, Yaoundé Central Hospital, Cameroon. Eligible participants were adult patients with diabetes $(n=12)$ spanning three strata of capillary glucose values $(100 \pm 40 \mathrm{mg} / \mathrm{dL}[5.55 \pm 2.22 \mathrm{mmol} / \mathrm{L}], 200 \pm 40 \mathrm{mg} / \mathrm{dL}$ $[11.1 \pm 2.22 \mathrm{mmol} / \mathrm{L}]$ and $300 \pm 40 \mathrm{mg} / \mathrm{dL}[16.65 \pm 2.22 \mathrm{mmol} / \mathrm{L}])$ and a control group $(n=4)$ of healthy normoglycaemic individuals (without diabetes). Individuals with long-term glucocorticoid use, patients with severe hyperglycaemia (hyperosmolar state, isolated ketosis or ketoacidosis), hypoglycaemia and/or coagulopathy were excluded.

\section{Study procedures}

In order to identify the influence of the studied body lotions, we measured capillary blood glucose before, immediately after application $\left(T_{0}\right)$, then 5 , 30 and 60 minutes after the application. We used three types of body lotions most frequently used in sub-Saharan Africa, and specifically in Cameroon, including sweet almond oil (4.8\% per $100 \mathrm{mg}$ carbohydrates, $80 \%$ oleic acid, $20 \%$ linoleic acid and other fatty acid), corticosteroid cream (0.1\% hydrocortisone 17 butyrate per $100 \mathrm{ml}$ ) and hydroquinone skin-lightening lotion (2.0\% hydroquinone per $100 \mathrm{ml}$ ). A swab prepared for 3 seconds by dipping in each body lotion was used to apply one thin layer on the distal phalanx of third and fourth fingers of the left and right hands of all participants. Reference values used to compare capillary measurements were plasma glucose and capillary glucose of clean finger (fifth finger of left hand after a careful hand washing). Capillary blood glucose was measured using a drop of whole capillary blood obtained by pricking a fingertip with a lancet and then placed on the reagent bonded to the strip, analysed by glucometer (Accu-Chek ${ }^{\circledR}$ Performa Roche, Mannheim, Germany), which uses glucose dehydrogenase enzyme to convert the glucose in the blood to gluconolactone in the presence of pyrroloquinoline quinone coenzyme. The coefficient of variation of the device was $<2 \%$ at the low, medium and high glucose levels. There was no difference between capillary glucose values before applications of sweet almond oil, corticosteroid cream or hydroquinone skin-lightening lotion compared to the clean finger. Therefore, we describe capillary glucose variations over time after application of the various cosmetics in comparison to the clean finger (control).

\section{Statistical analysis}

Data were analysed using Stata 14.0 for Windows (StataCorp, TX, US). Figures were represented using GraphPad Prism version 7.00 for Windows (GraphPad, San Diego, CA, US). Wilcoxon sign rank or KruskalWallis test was used to explore the glycaemia before and after body Iotion administration. The magnitude of the difference from baseline was further assessed using the corrected variation of capillary glucose from baseline values. This difference was expressed as percentage of the baseline capillary glucose value. A probability of $p<0.05$ was set as the threshold of statistical significance.

\section{Results}

The mean age in our study was $41.68 \pm 15.80$ years, and nine out of the 16 participants were women. The mean value for capillary glycaemia of clean finger was $244 \pm 161 \mathrm{mg} / \mathrm{dL}(13.54 \pm 8.94 \mathrm{mmol} / \mathrm{L})$.

Unlike the corticoid cream and sweet almond oil (delta [95\% Cl]: $-0.2 \%$ $[-7.9-2.6]$ and $0.5[-5.7-6.9]$ respectively, both $p$-values $>0.05)$, there was
Figure 1: Comparison of capillary blood glucose measurement and dynamic of capillary glucose over time

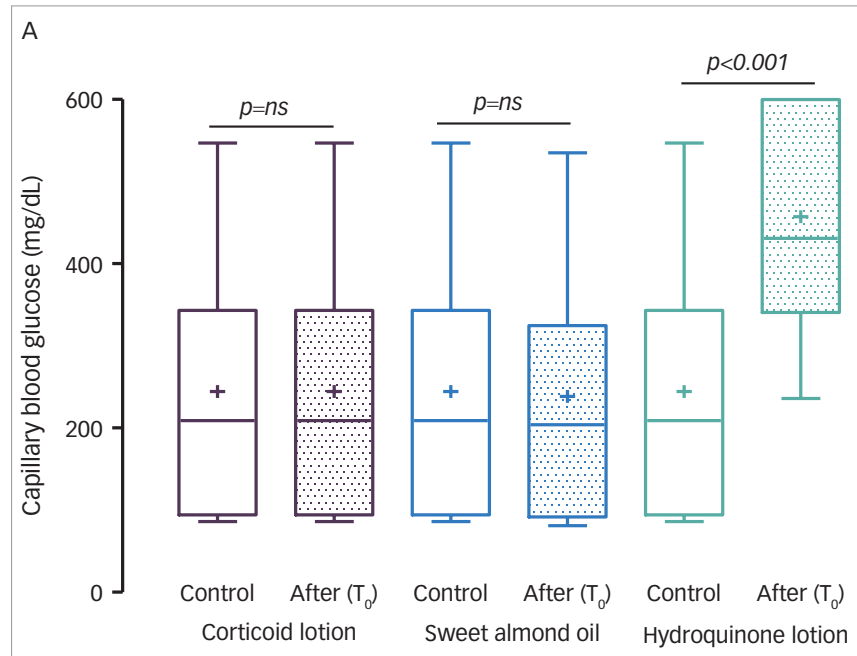

B



** $p<0.001 ; * p<0.05$. Comparison of capillary blood glucose between clean finger (control) and immediately after administration of body lotions (A), and dynamic of capillary glucose over time $(0,5,30$ and 60 minutes) after administration of corticoid lotion, sweet almond oil and hydroquinone-containing body lotion (B). $T_{0}=$ administration.

a significant increase (delta [95\% Cl]: 119.5\% [77.4-222.1]) of capillary glucose level immediately after administration $\left(T_{0}\right)$ of hydroquinonecontaining body lotion $(p<0.001)$ (Figure $1 A)$. Capillary glucose values after the use of corticoid cream ( $p=0.873$ ) and sweet almond oil $(p=0.883$ ) was stable over time (5, 30 and 60 minutes after application). In the case of the hydroquinone-containing body lotion, there was a significant difference between capillary glucose level 5 minutes (delta [95\% Cl]: 81.6\% [55.3-214.2]; $p<0.001), 30$ minutes (delta [95\% Cl]: 71.6\% [21.8134.6]; $p<0.001$ ) and up to 60 minutes (delta [95\% Cl]: 58.3\% [2.8-133.3]; $\mathrm{p}=0.013$ ), after administration compared to the value obtained from the clean finger (Figure 1B).

\section{Discussion}

The body lotions used for this study are the most commonly commercialised ones in Cameroon and in many other sub-Saharan African countries. Skin-lightening is a very common practice in sub-Saharan African populations especially among women; for this purpose, hydroquinone and corticosteroids are the most commonly used cosmetics. A number of complications associated to the use of these products has been previously reported and include local and systemic complications..$^{10}$ In this study, we focused on the immediate effects of sweet almond oil, a corticosteroid 
cream and a hydroquinone-containing skin-lightening lotion on the accuracy of capillary glucose measurement over time.

We found that capillary blood glucose obtained after application of sweet almond oil and corticoid cream compared to clean finger showed no significant difference. These results were similar to those obtained by Ginsberg who found that there was no difference in capillary blood glucose from fingers soaked with a moisturising lotion rich in sweet almond compared to clean fingers. ${ }^{6}$ The use of the hydroquinone-containing body lotion on the other hand significantly increased the measured value of capillary glucose level. This overestimation was persistent up to 1 hour after application of this latter cosmetic. A similar observation has been reported by Bouche et al. after the use of a 1\% hydroquinone-containing body lotion, half the concentration used in our study. ${ }^{9}$ Bouche et al. observed discrepant capillary glycaemic values in two insulin-treated patients with diabetes. An elevated capillary glucose was observed in the first case despite previous insulin adjustments in the absence of glycosuria. In the second case, a discrepancy was obtained with a clinical presentation suggestive of hypoglycaemia. This shows the clinical relevance of capillary glucose measurements which could be affected by inaccurate readings related to the use of hydroquinonecontaining body lotions. Furthermore, one could easily imagine the negative impact of falsely elevated values on patients' management with an accretion of the risk of hypoglycaemia in patients treated with CSII or MDI. ${ }^{11}$ Furthermore, this could yield discrepant results in patients under continuous glucose monitoring devices requiring calibration or verification, at high or low interstitial glucose levels, with capillary glucose measurements.
Our study has the merit of enrolling participants in a broad range of glycaemia with the aim to evaluate the influence of the three commonly used body lotions in sub-Saharan Africa. It is, however, likely that the margin of error (delta) from the baseline glucose value, used to estimate deviation from the value obtained from the clean finger, would be smaller in high glucose range. We therefore acknowledge that the limited number of participants per glucose strata does not enable us to further explore the impact of the explored body lotions at the different levels of glucose values. It is obvious however that as opposed to the patients with very high capillary glucose value, falsely elevated capillary glucose could likely lead to more clinically relevant deleterious consequences in patients in the medium or low glucose level $(75 \%$ of our study population). In this range of glucose values, falsely elevated glucose measurements could eventually induce an additional challenge to the diagnosis of hypoglycaemic episodes especially in patients with problematic hypoglycaemia. ${ }^{12}$ Hand washing prior to capillary glucose measurement should therefore strongly be recommended to patients with diabetes mellitus.?

\section{Conclusion}

Hydroquinone-containing body lotions induce falsely elevated capillary glucose measurements that persisted up to 60 minutes after usage. Education of both health practitioners and patients on this potential influence of hydroquinone-containing cosmetics could reduce misinterpretation of inappropriately high capillary glucose levels in daily clinical practice. $\square$
1. American Diabetes Association. Standards of Medical Care in Diabetes-2016: Summary of Revisions. Diabetes Care. 2016;39:S4-5.

2. Huang IC, Wang PW, Liu RT, et al. The influence of self-monitoring blood glucose frequency on the oscillation of hemoglobin A1c and chronic complications. Chang Gung Med J. 2012;35:46-53

3. Hortensius J, Kars MC, Wierenga WS, et al. Perspectives of patients with type 1 or insulin-treated type 2 diabetes on self-monitoring of blood glucose: a qualitative study. BMC Public Health. 2012;12:167.

4. Murata T, Tsuzaki K, Yoshioka F, et al. The relationship between the frequency of self-monitoring of blood glucose and glycaemic control in patients with type 1 diabetes mellitus on continuous subcutaneous insulin infusion or on multiple daily injections. J Diabetes Investig. 2015;6:687-91. 5. Rebel A, Rice MA, Fahy BG. Accuracy of point-of-care glucose

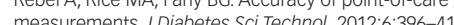

6. Ginsberg BH. Factors affecting blood glucose monitoring sources of errors in measuremt. Diabetes Sci Techno: 2009;3:903-13

7. Veigne SW, Sobngwi E, Nouthe BE, et al. Study of the effect of altitude on the measurement of glycated haemoglobin using point-of-care instruments. Cardiovasc J Afr. 2015;26:38-40.

8. Hirose T, Mita T, Fujitani Y, et al. Glucose monitoring after fruit peeling: pseudohyperglycaemia when neglecting hand washing before fingertip blood sampling: wash your hands with tap water before you check blood glucose level. Diabetes Care. 2011:34:596-7.
9. Bouche $\mathrm{CH}$, Garnier JP, Choukem SP, Gautier JF. Falsely elevated capillary glucose and ketone levels and use of skin lightening creams. BMJ. 2015;351 h3879. Skin lightening creams. BM.

10. Olumide $\mathrm{YM}$, Akinkugbe $\mathrm{AO}$, Altraide $\mathrm{D}$, et al. Complications of chronic use of skin lightening. Int I Dermatol. of chronic use of

11. Juvenile Diabetes Research Foundation Continuous Glucose Monitoring Study Group, Tamborlane WV, Beck RW, et al. continuous glucose monitoring and intensive treatment of type 1 diabetes. N Eng/ J Med. 2008;359:1464-76.

12. Choudhary P, Rickels MR, Senior PA, et al. Evidence-informed clinical practice recommendations for treatment of type 1 diabetes complicated by problematic hypoglycemia. Diabetes Care. 2015;38:1016-29. 